\title{
Tuning molecular motor transport through cytoskeletal filament network organization
}

\author{
Monika Scholz, ${ }^{1,2}$ Kimberly L. Weirich, ${ }^{1,2}$ Margaret L. Gardel, ${ }^{1,2,3}$ and Aaron R. Dinner ${ }^{1,2,4, *}$ \\ ${ }^{1}$ James Franck Institute, the University of Chicago, Chicago, IL 60637 \\ ${ }^{2}$ Institute for Biophysical Dynamics, the University of Chicago, Chicago, IL 60637 \\ ${ }^{3}$ Department of Physics, the University of Chicago, Chicago, IL 60637 \\ ${ }^{4}$ Department of Chemistry, the University of Chicago, Chicago, IL 6063r*
}

\begin{abstract}
The interaction of motor proteins with intracellular filaments is required for transport processes and force generation in cells. Within a cell, crosslinking proteins organize cytoskeletal filaments both temporally and spatially to create dynamic, and structurally diverse networks. The architecture of these networks changes both the mechanics as well as the transport dynamics; however, the effects on transport are less well understood. Here, we compare the transport dynamics of myosin II motor proteins moving on model cytoskeletal networks created by common crosslinking proteins. We observe that motor dynamics change predictably based on the microstructure of the underlying networks and discuss how this can be utilized by cells to achieve specific transport goals.
\end{abstract}

\section{INTRODUCTION}

The interior of cells is organized by cytoskeletal structures. Protein monomers like actin and tubulin organize into polymers, resulting in polar filaments that can span cellular dimensions. These filaments interact with molecular motor proteins to drive intracellular transport, cellular shape change, and cell motility [1]. These different functions rely on different arrangements of the filaments, ranging from tight bundles in filopodia to meshes in the cell cortex [2].

Filaments are organized locally by proteins that can bind to multiple actin filaments to crosslink them into a network [2]. The type of crosslinking protein determines the resulting network structure. Proteins with a small crosslinking distance, such as fimbrin or $\alpha$-actinin, create tightly packed bundles with the filaments spaced 8-36 nm apart $[3,4]$. The crosslinking protein fascin creates similarly tight bundles, but the actin filaments are polaritysorted as well $[5,6]$. In contrast, filamin has a crosslinking distance of $160 \mathrm{~nm}$, resulting in a loosely bundled structure for low concentrations of crosslinking protein [7]. While the consequences of these diverse structures for network mechanics have the focus of several studies [813], the consequences for motor transport have received less attention $[14,15]$.

We recently found that the microscopic arrangement of filaments can have profound effects on the transport of motor proteins across filament networks [16]. In particular, the presence of filament loops can lead to unproductive cycling of motors. These vortex-like states give rise to a power-law distribution of dwell times and glassy dynamics in intracellular transport. We showed that we could tune the transport properties of the motors by varying properties of the system that control the number of junctions that can support cycling. We thus predicted that cells could modulate their cytoskeletal struc-

\footnotetext{
* To whom correspondence may be addressed: dinner@uchicago.edu
}

tures spatially and temporally to control the motor dynamics.

Here, we demonstrate experimentally that actin filament network organization can modulate transport dynamics of myosin II motors. To isolate the effects associated with bundle architecture from those stemming from cellular regulation [17], we track myosin II on model networks of actin filaments bundled by biological crosslinking proteins. The dynamics can be interpreted in terms of the known features of the crosslinking proteins.

\section{TRANSPORT PROPERTIES DEPEND ON THE MICROSCOPIC BUNDLE ARCHITECTURE}

We analyze single-particle tracking data from myosin II motors moving on a quasi-two-dimensional (quasi2D) actin network (Fig. 1). The actin filaments are crosslinked with either $\alpha$-actinin, fimbrin, fascin or filamin to create actin bundles with distinct microstructures (Fig. 1). The actin networks and skeletal-muscle myosin II were prepared as described in [16]. Singleparticle trajectories were obtained using the Pythonbased implementation of the Crocker-Grier algorithm Trackpy [18]. The resulting trajectories align well with the crosslinked actin filaments (Fig. 1).

For each experimental condition, we characterize the motor motion through the time-averaged mean-squared displacement (TA-MSD) as a function of lag time $\Delta$ and measurement time $T$ as previously described $[16,19,20]$ :

$$
\left\langle\overline{\vec{R}(T, \Delta)^{2}}\right\rangle=\frac{1}{T-\Delta} \int_{0}^{T-\Delta}[\vec{x}(t+\Delta)-\vec{x}(t)]^{2} d t,
$$

where $\vec{x}(t)$ is the position of a motor at time $t$.

The exponent $\alpha$ in the scaling relation $\left\langle\overline{\vec{R}(T, \Delta)^{2}}\right\rangle \propto$ $\Delta^{\alpha}$ characterizes the motion: $\alpha=1$ for simple diffusion, and $\alpha=2$ for a purely inertial motion; non-integer values are possible as well (e.g., $[19,20]$ and references therein). We observe that the exponent of the TA-MSD as a function of lag time changes depending in the underlying bundle structure (Fig. 2). The polarity-sorted 

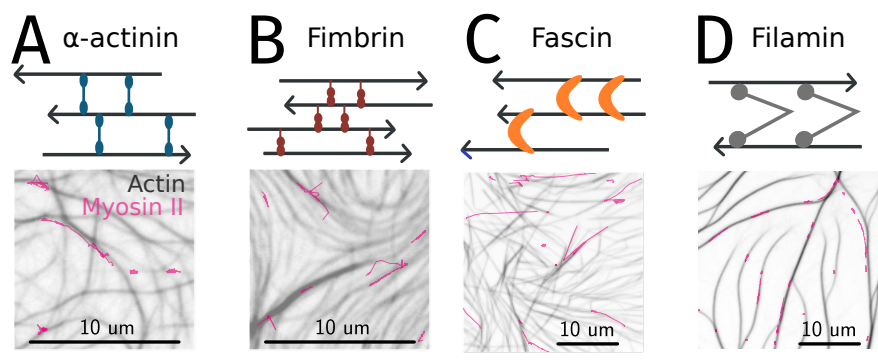

FIG. 1. Schematic showing the interactions of four crosslinking proteins with actin filaments (red arrows). Fluorescence microscopy image of the quasi-2D actin networks after crosslinking with either $\alpha$-actinin (A), fimbrin (B), fascin (C) or filamin (D). The trajectories of myosin II are overlaid (red). Note that the scale of the images change between panels, due to the large difference in myosin II speeds on different crosslinked actin networks.

bundles created by the crosslinking protein fascin lead to an exponent close to two, indicating strongly directed motion. In contrast, the exponent of the TA-MSD is closer to one for filamin, $\alpha$-actinin and fimbrin.

We also consider the TA-MSD as a function of measurement time, $T$. This order parameter is sensitive to the ergodicity of the dynamics. If the properties of the transport process underlying the observed dynamics are unchanged over the course of measurement, the dynamics are ergodic, and the expected exponent is zero. However, as previously described for a fimbrin-crosslinked network, the mean-square displacement decreases as the measurement time increases (i.e., the exponent is less than zero) [16]. We find similar exponents for both $\alpha$-actinin and fimbrin, which is unexpected considering the relevant scales. We previously described a mechanism for these non-ergodic dynamics that relies on motors interacting with at least two filaments at the same time [16] and getting effectively trapped. Filaments in fimbrin bundles are spaced closer than the binding radius of a myosin motor, and the myosin minifilament can interact with many filaments at the same time, supporting non-ergodic dynamics. However, the filament spacing in $\alpha$-actinin bundles is larger than the myosin binding radius, which would suggest that the myosin minifilament spans these gaps via its long axis of $100-500 \mathrm{~nm}$, which is considerably larger than the bundle spacing. The exponents for filamin and fascin do not deviate as strongly from zero, indicating that the dynamics are only weakly non-ergodic. In the case of fascin, the unipolar bundles favor directional transport, and do not support trapping, even if the motor protein interacts with multiple filaments at once. In the case of filamin the large filament spacing often leads to the motor protein interacting with a single filament.

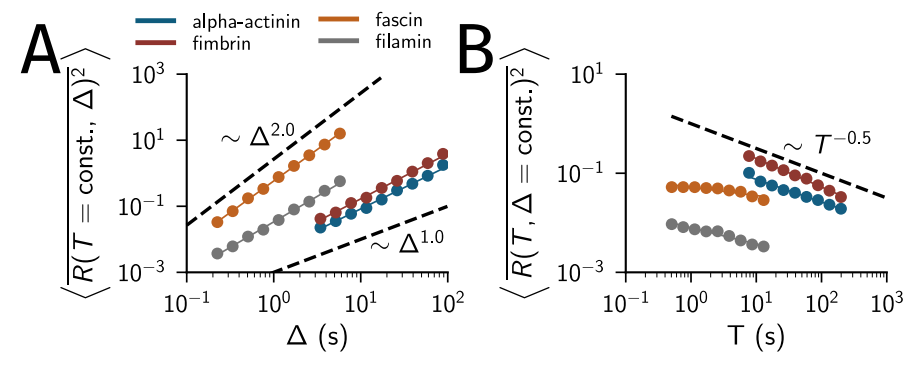

FIG. 2. (A) Mean-squared displacement of myosin II minifilaments on actin networks crosslinked with different proteins as a function of lag time $(\Delta)$ and $(\mathrm{B})$ as a function of measurement time $(T) . T=9 \mathrm{~s}$ and $\Delta=0.2 \mathrm{~s}$ for fascin (orange) and filamin (gray). For $\alpha$-actinin (blue) and fimbrin (red), $T=137.7 \mathrm{~s}$ and $\Delta=3.06 \mathrm{~s}$. The number of trajectories is $210,251,236$ and 256 for $\alpha$-actinin, fimbrin, fascin and filamin, respectively. The mean trajectory lengths are 167, 208, 16 and $17 \mathrm{~s}$ for $\alpha$-actinin, fimbrin, fascin and filamin, respectively.

\section{ANGULAR DISTRIBUTION ILLUSTRATES MICROSCOPIC DIRECTIONAL CHANGES}

The TA-MSD cannot reveal directional correlations within trajectories, but there is evidence of such correlations in particle-tracking studies of molecular motors [19-24]. To quantify such correlations, we use the relative angle distribution [20]. The relative angle is defined by

$$
\cos \theta(t ; \Delta)=\frac{\vec{v}(t ; \Delta) \cdot \vec{v}(t+\Delta ; \Delta)}{|\vec{v}(t ; \Delta)||\vec{v}(t+\Delta ; \Delta)|},
$$

where $\vec{v}(t ; \Delta)=\vec{x}(t+\Delta)-\vec{x}(t)$. The normalized histogram (probability density function, PDF) of $\theta$ values for successive vectors within trajectories can be used as a directional order parameter.

The relative angle distribution is flat for simple diffusion because there are no correlations between steps of the random walk. A dictionary of relative angle distributions for a variety of more complicated transport processes can be found in [20]. Consistent with previous observations for molecular motors [20], for the four experimental conditions that we consider here (Fig. 3), we generally observe peaks at $\theta=0$ and $\theta=\pi$, indicating an apparently inertial motion and frequent reversals, respectively.

The relative angle distribution can be calculated at different $\Delta$ to elucidate the timescales contributing to the motion. To investigate the effects on the transport of local structure, as opposed to large-scale network topology, we chose a small $\Delta(\Delta=0.1,0.1,1.53,1.53 \mathrm{~s}$ for fascin, filamin $\alpha$-actinin and fimbrin, respectively). To compare recordings of the motion on different crosslinked networks, the magnitude of $\Delta$ was chosen to be inversely proportional to the mean motor velocity on a given network. For example, the mean velocity of myosin on the fimbrin-crosslinked network is only $38 \mathrm{~nm} / \mathrm{s}$ whereas mo- 
tors on the fascin-crosslinked network move at $620 \mathrm{~nm} / \mathrm{s}$. Thus, the respective values for $\Delta$ are $1.53 \mathrm{~s}$ and $0.1 \mathrm{~s}$, respectively. We include all trajectories in the analysis, even those that are not moving significantly during our measurement. The average motor velocity on fascin bundles is smaller than the unloaded gliding speed of myosin on actin filaments $[25,26]$.

The motors on the fimbrin-crosslinked network show a strong peak at $\pi$, indicating that motors change direction frequently. This is consistent with tight, mixed polarity bundles that create an environment that supports tugof-war [27, 28] and cycling [16] mechanisms. Again the results for $\alpha$-actinin and fimbrin are similar, consistent with a trapping between the tighter bundles along the long axis of the myosin minifilament in both of these bundles. The relative angle distribution for $\alpha$-actinin also agrees with a previously reported data set [20].

In contrast, the motor transport on fascin-crosslinked bundles shows a strong directional component, as evidenced by the peak at $\theta=0$ in the relative angle distribution. Since fascin arranges actin filaments into polarity sorted bundles, this is likely due to directed movement along a bundle. The loose bundles created by filamin result in a nearly flat angular distribution with only small peaks at $\theta=0$ and $\pi$. This indicates transport dominated by diffusion with only small directional components and few reversals.

Interestingly, the time-averaged mean-squared displacement shows a super-diffusive behavior for transport on the filamin-crosslinked network (Fig. 2A). Therefore, over large timescales the motor exhibits directed motion along the filamin network. However, on the smaller timescale used to calculate the angular distribution the motor shows nearly diffusional behavior. This could support two hypotheses. One is that the motors frequently hop between filaments in different microscopic orientations, such that the motion resembles a biased random walk. The other possible hypothesis is that the motors are detaching and diffusing within the bundles. Taken together, these measurements show that the relative angle distribution is sensitive to the bundle structure and can distinguish bundle structures resulting from different crosslinking proteins.

\section{DISCUSSION}

Using motor trajectories obtained from tracking myosin II motors moving on reconstitued actin networks, we find that transport depends on the microscopic structure of the filament network. Intuitively, polarity-sorted bundle structures lead to directional transport, whereas mixed polarity bundles result in a mixture of directed motion and trapped motion. Since the motor complex has a finite "reach", the loose bundles formed by filamin are less effective at trapping the motor than the tightly spaced fimbrin bundles. From our results, one can deduce the dynamics on a hypothetical actin network with

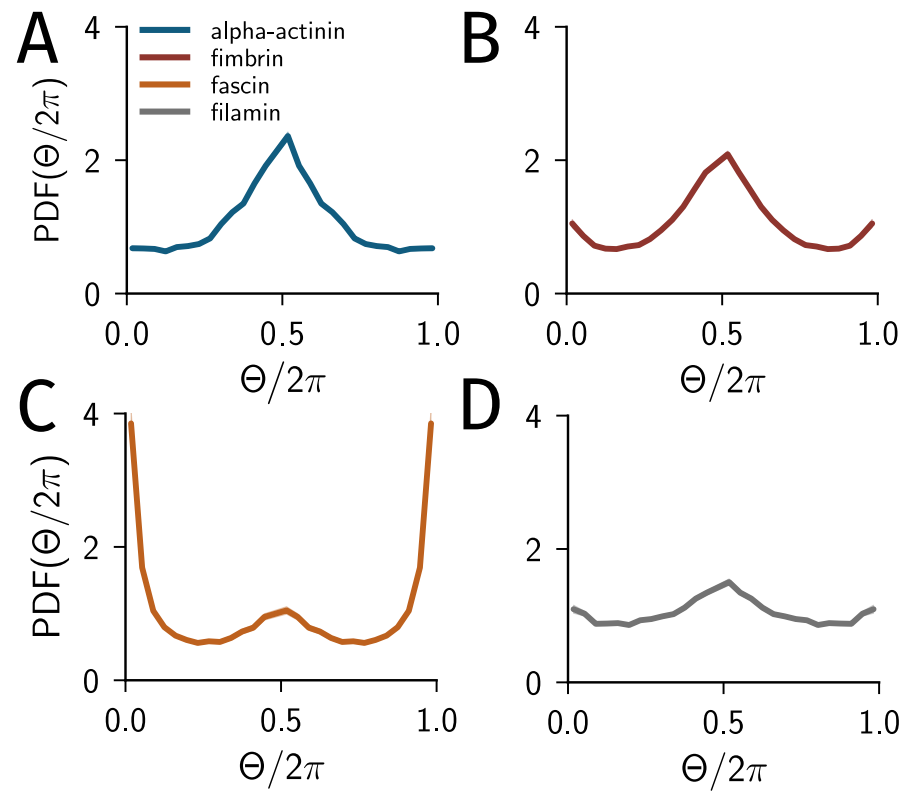

FIG. 3. Relative angle distributions of myosin II motor trajectories on actin networks crosslinked with either $\alpha$-actinin (A), fimbrin (B), fascin (C) or filamin (D). See text for discussion of the choice of $\Delta$.

wide filament spacing but polarity-sorted bundles. Our results would suggest that the resulting motion would have a strong forward-directed component, but no significant trapping, thus resulting in apparently inertial dynamics over short timescales and simple diffusion over long timescales.

Our results have implications for the regulation of transport in cells, but is especially relevant for secreting cells, such as pancreatic beta cells. Although traditionally associated with microtubule-based transport, insulin secretory vesicles in beta cells have to cross the actin cortex before fusing with the membrane to release the hormone into the bloodstream [19]. The actin cortex is remodeled in response to insulin signaling [29]. It is likely that the timing of insulin release is affected by the structure of the actin cortex, and that the reported actin remodelling serves the purpose of making the network more amenable for traversing granules [30].

In summary, our results show that the organization of cytoskeletal networks has not only mechanical, but also dynamical consequences. The prevalence of different crosslinking proteins in different cellular regions, such as fillopodia, lamellopodia, or the cell cortex, could also be optimized for the transport processes that occur in those regions. Further studies should investigate the role of crosslinking proteins on motor dynamics in vivo. The principles that we elucidated could also be exploited to design novel materials with defined transport properties. 


\begin{tabular}{ll}
\hline Crosslinker & Exponent $\left\langle\overrightarrow{\left.\vec{R}(T=\text { const., } \Delta)^{2}\right\rangle}\right.$ \\
\hline$\alpha$-actinin & $1.293 \pm 0.036$ \\
fimbrin & $1.369 \pm 0.034$ \\
fascin & $1.905 \pm 0.016$ \\
filamin & $1.543 \pm 0.033$ \\
\hline & \\
\hline Crosslinker & Exponent $\left\langle\overrightarrow{\vec{R}(T, \Delta=\text { const })^{2}}\right\rangle$ \\
\hline$\alpha$-actinin & $-0.457 \pm 0.022$ \\
fimbrin & $-0.573 \pm 0.018$ \\
fascin & $-0.168 \pm 0.034$ \\
filamin & $-0.329 \pm 0.027$ \\
\hline
\end{tabular}

\section{ACKNOWLEDGMENTS}

We thank Stanislav Burov for helpful discussions. We thank Samantha Stam, Todd Thoresen, and members of David Kovar's laboratory (Jenna Christensen, Jonathon Winkelmann, and Youjii Li) for purified proteins. This work was primarily supported by the University of Chicago Materials Research Science and Engineering Center under award NSF DMR-1420709.

TABLE I. Exponents of the mean-squared displacement shown in Fig. 2. The error is the statistical error of the fit.

[1] Laurent Blanchoin, Rajaa Boujemaa-Paterski, Cécile Sykes, and Julie Plastino, "Actin dynamics, architecture, and mechanics in cell motility," Physiological reviews 94, 235-263 (2014).

[2] Ekta Seth Chhabra and Henry N Higgs, "The many faces of actin: matching assembly factors with cellular structures," Nature cell biology 9, 1110-1121 (2007).

[3] Kenneth A Taylor and Dianne W Taylor, "Formation of two-dimensional complexes of f-actin and crosslinking proteins on lipid monolayers: demonstration of unipolar alpha-actinin-f-actin crosslinking," Biophysical journal 67, 1976-1983 (1994).

[4] Anthony Bretscher, "Fimbrin is a cytoskeletal protein that crosslinks f-actin in vitro," Proceedings of the National Academy of Sciences 78, 6849-6853 (1981).

[5] James R Bartles, "Parallel actin bundles and their multiple actin-bundling proteins," Current opinion in cell biology 12, 72-78 (2000).

[6] Jonathan D Winkelman, Cristian Suarez, Glen M Hocky, Alyssa J Harker, Alisha N Morganthaler, Jenna R Christensen, Gregory A Voth, James R Bartles, and David R Kovar, "Fascin-and $\alpha$-actinin-bundled networks contain intrinsic structural features that drive protein sorting," Current Biology 26, 2697-2706 (2016).

[7] Fumihiko Nakamura, Thomas P Stossel, and John H Hartwig, "The filamins: organizers of cell structure and function," Cell adhesion \& migration 5, 160-169 (2011).

[8] Gijsje H Koenderink and Ewa K Paluch, "Architecture shapes contractility in actomyosin networks," Current Opinion in Cell Biology 50, 79-85 (2018).

[9] Elizabeth M Reichl, Yixin Ren, Mary K Morphew, Michael Delannoy, Janet C Effler, Kristine D Girard, Srikanth Divi, Pablo A Iglesias, Scot C Kuo, and Douglas N Robinson, "Interactions between myosin and actin crosslinkers control cytokinesis contractility dynamics and mechanics," Current Biology 18, 471-480 (2008).

[10] KM Schmoller, O Lieleg, and AR Bausch, "Crosslinking molecules modify composite actin networks independently," Physical review letters 101, 118102 (2008).

[11] Oliver Lieleg, Mireille MAE Claessens, and Andreas R Bausch, "Structure and dynamics of cross-linked actin networks," Soft Matter 6, 218-225 (2010).

[12] Thomas P Stossel, John Condeelis, Lynn Cooley, John H Hartwig, Angelika Noegel, Michael Schleicher, and Sandor S Shapiro, "Filamins as integrators of cell mechanics and signalling," Nature reviews Molecular cell biology 2, 138 (2001).

[13] Jonathan Stricker, Tobias Falzone, and Margaret L Gardel, "Mechanics of the f-actin cytoskeleton," Journal of biomechanics 43, 9-14 (2010).

[14] Rüdiger Rudolf, Thorsten Salm, Amin Rustom, and Hans-Hermann Gerdes, "Dynamics of immature secretory granules: role of cytoskeletal elements during transport, cortical restriction, and f-actin-dependent tethering," Molecular Biology of the Cell 12, 1353-1365 (2001).

[15] Martin Oheim and Walter Stühmer, "Tracking chromaffin granules on their way through the actin cortex," European Biophysics Journal 29, 67-89 (2000).

[16] Monika Scholz, Stanislav Burov, Kimberly L Weirich, Björn J Scholz, SM Ali Tabei, Margaret L Gardel, and Aaron R Dinner, "Cycling state that can lead to glassy dynamics in intracellular transport," Physical Review X 6, 011037 (2016).

[17] Michael P Murrell and Margaret L Gardel, "F-actin buckling coordinates contractility and severing in a biomimetic actomyosin cortex," Proc. Natl. Acad. Sci. USA 109, 20820-20825 (2012).

[18] Daniel B. Allan, Thomas A. Caswell, and Nathan C. Keim, "Trackpy v0.2," (2014).

[19] SM Ali Tabei, Stanislav Burov, Hee Y Kim, Andrey Kuznetsov, Toan Huynh, Justin Jureller, Louis H Philipson, Aaron R Dinner, and Norbert F Scherer, "Intracellular transport of insulin granules is a subordinated random walk," Proceedings of the National Academy of Sciences 110, 4911-4916 (2013).

[20] Stanislav Burov, SM Ali Tabei, Toan Huynh, Michael P Murrell, Louis H Philipson, Stuart A Rice, Margaret L Gardel, Norbert F Scherer, and Aaron R Dinner, "Distribution of directional change as a signature of complex dynamics," Proc. Natl. Acad. Sci. USA 110, 19689-19694 (2013). 
[21] I. Golding and E. C. Cox, "Physical Nature of Bacterial Cytoplasm," Phys. Rev. Lett. 96, 098102 (2006).

[22] Tolić-Norrelykke, Iva Marija and Munteanu, EmiliaLaura and Thon, Genevieve and Oddershede, Lene and Berg-Sorensen, Kirstine, "Anomalous diffusion in living yeast cells," Phys. Rev. Lett. 93, 078102 (2004).

[23] S.C. Weber, A.J. Spakowitz, and J.A. Theriot, "Bacterial Chromosomal Loci Move Subdiffusively through a Viscoelastic Cytoplasm," Phys. Rev. Lett. 104, 238102 (2010).

[24] A.V. Weigel, B. Simon, M.M. Tamkun, and D. Krapf, "Ergodic and nonergodic processes coexist in the plasma membrane as observed by single-molecule tracking," Proc. Natl. Acad. Sci. USA 108, 6438 (2011).

[25] James A Spudich, Stephen J Kron, and Michael P Sheetz, "Movement of myosin-coated beads on oriented filaments reconstituted from purified actin," Nature 315, 584-586 (1985).

[26] Stephen J Kron and James A Spudich, "Fluorescent actin filaments move on myosin fixed to a glass surface," Pro- ceedings of the National Academy of Sciences 83, 62726276 (1986).

[27] Melanie JI Müller, Stefan Klumpp, and Reinhard Lipowsky, "Tug-of-war as a cooperative mechanism for bidirectional cargo transport by molecular motors," Proc. Natl. Acad. Sci. USA 105, 4609-4614 (2008).

[28] William O Hancock, "Bidirectional cargo transport: moving beyond tug of war," Nat. Rev. Mol. Cell Biol. 15, 615-628 (2014).

[29] Ragadeepthi Tunduguru, Tim T Chiu, Latha Ramalingam, Jeffrey S Elmendorf, Amira Klip, and Debbie C Thurmond, "Signaling of the p21-activated kinase (pak1) coordinates insulin-stimulated actin remodeling and glucose uptake in skeletal muscle cells," Biochemical pharmacology 92, 380-388 (2014).

[30] Caroline Arous and Philippe A Halban, "The skeleton in the closet: actin cytoskeletal remodeling in $\beta$-cell function," American Journal of Physiology-Endocrinology and Metabolism 309, E611-E620 (2015). 